\title{
LA ARQUITECTURA POPULAR COMO BASE DE UNA ARQUITECTURA BIOCLIMATICA. APLICACION AL ENFRIAMIENTO PASIVO
}

\author{
(THE POPULAR ARCHITECTURE BASE FOR A CLIMATIC ARCHITECTURE. APPLICATION TO \\ PASSIVE COOLING)
}

\author{
J. L. Esteban Sáiz. Dr. Ingeniero Industrial \\ IETCC/CSIC
}

RESUMEN

En este articulo se describe la diversidad arquitectónica popular que puede apreciarse en España debido, en parte, a la gran influencia climatológica de las diferentes regiones. Se hace resaltar la indudable similitud con otros paises, de condiciones ambientales análogas, independientemente de las relaciones que hayan existido entre sus moradores.

La entrada de tendencias innovadoras en la edificación ha requerido investigar sobre nuevos diseños constructivos adecuados a las condiciones ambientales y considerando conceptos termofísicos, que hasta el momento actual no se utilizaban, hasta llegar a una arquitectura que pudiéramos llamar bioclimática.

\section{SUMMARY}

In this work it is described de diversity of popular architectures that can be found in Spain due to the strong climatological influence in the different zones of the country. It is also pointed out their similarity with the architecture of other countries, independently of the connexions existing between their dwellers.

The rise of new tendencies in construction has brought the need to investigate on new architectural design adapted to ambient conditions considering thermophysical conceipts, which till now were not used, since a so called bioclimatic architecture woulbe be reached.

\section{INTRODUCCION}

El ser humano tiende a crear en torno a él unas condiciones ambientales lo más satisfactorias posible, de forma que pueda desarrollar su actividad dentro de un confort higrotérmico adecuado.

Indudablemente los distintos indices térmicos que combinan la temperatura, humedad y velocidad del viento, fijados por diferentes autores (1), definen diversos parámetros, tales como temperatura efectiva: Predicted Mean Vote (P.M.V., Predicted Percentage of Dissatisfied (P.P.D.), etc., que sirven para determinar un grado de confort de acuerdo con las condiciones climáticas donde se desenvuelven los individuos, asi como su propio metabolismo; por lo tanto, lo interesante será conseguir que un elevado porcentaje de los ocupantes se encuentren en condiciones higrotérmicas satisfactorias, tal como indica la norma ASHRAE 55-81, que sitúa este valor en el $85 \%(2)$.

\section{ARQUITECTURA POPULAR}

Este afán de confort ambiental ha sido constante desde las antiguas civilizaciones, en el transcurso del tiempo, para lo cual los antiguos moradores fueron creando unos "hábitats" caracteristicos a las distintas condiciones climáticas a que habian estado sometidos y que, paulatinamente, año tras año, han ido modificando. Dichas primitivas construcciones se fueron acoplando con los recursos comunes de las zonas, más próximas, a un tipo de arquitectura regional acorde con las caracteristicas ambientales, obteniendo de éstas una gran rentabilidad para, de esta forma, poder tener en el interior de la vivienda unas condiciones ambientales lo más próximas a las del confort.

Como ejemplo, de lo dicho anteriormente, es interesante resaltar la diversidad arquitectónica popular que puede apreciarse en España, si bien la influencia tan importante que tiene el clima nos lleva a poder apreciar, en otros 


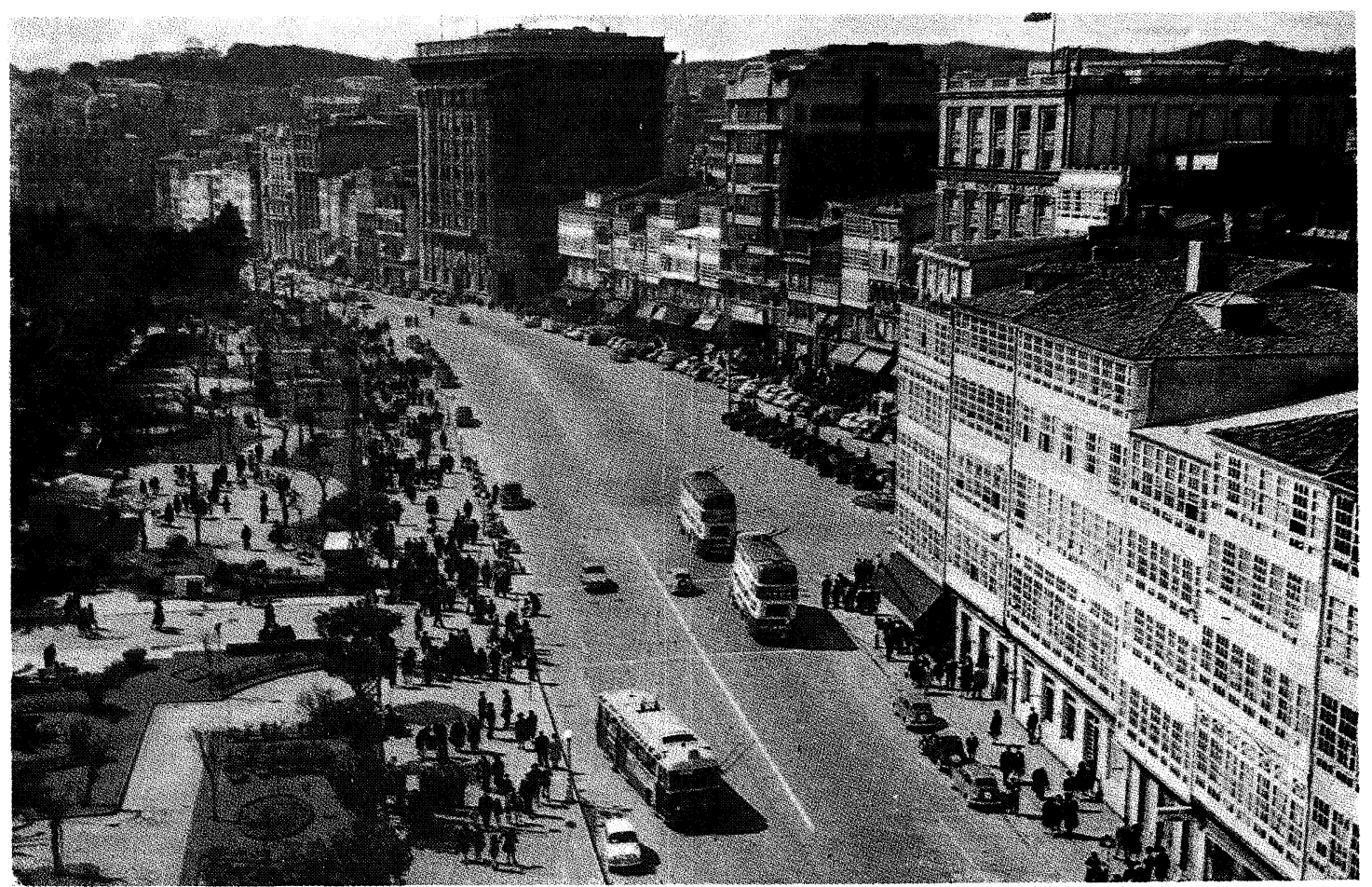

Foto 1

lugares de condiciones ambientales análogas, una arquitectura que, en sus partes fundamentales de tratamiento de los agentes externos, tiene gran similitud con la generada por nuestros antepasados, es decir, que independientemente de las relaciones que hayan existido entre los pobladores de los distintos paises, cada pueblo ha elegido y desarrollado una forma de diseño arquitectónico que es función principalmente del clima y que, en muchos casos, tiene gran concordancia. Como ejemplo más significativo puede indicarse la arquitectura mediterránea, donde muchas viviendas de caracteristicas populares, de paises como Túnez, Grecia o de la misma Andalucia, ofrecen tal similitud que, en un hipotético cambio de ubicación entre sí, quedarian perfectamiente integrados con su entorno popular.

En un paseo por nuestra Geografia podemos admirar la variedad arquitectónica creada por el propio pueblo que se nos ofrece: desde la tendencia, en las zonas más al norte, de una mejor captación solar de forma directa o indirecta, mediante el efecto de invernadero y acumulación calorifica por el propio cerramiento, hasta las zonas situadas más al sur, donde la tendencia en general es evitar la gran radiación solar y provocar un mayor enfriamiento interior. Es decir, se tienen dos zonas totalmente diferenciadas como punto extremo de un abanico de múltiples posibilidades; asi, cuando en la zona norte las condiciones de confort se aproximan más a la época de verano, en las zonas situadas al sur esta situación es a la inversa y está más próxima al invierno, luego las acciones llevadas a cabo bajo el punto el vista arquitectónico, para adecuarlas a unas condiciones higrotérmicas óptimas, han sido totalmente diferentes.

\subsection{Sistemas característicos}

Como puntos más notables pudriamos destacar, dentro de la zona norte, la arquitectura gallega, y corno diseño más característico el sistema de galerias acristaladas que posteriormente fue trasladé. - tose a otros muchos puntos del litoral cantábrıco.

En la fotografia n.o 1 puede apreciarse el Paseo de la Marina, de La Coruña, con los sistemas de acristalamiento prácticamente corridos de un edificio a otro, cuyo origen parece ser que se remonta al siglo XVIII, donde se efectúa una ganancia directa, asi como indirecta, mediante el efecto invernadero, con acumulación de calor, principalmente en el interior de la vivienda a través del suelo o los tabiques interiores, en los que inciden directamente los rayos solares y, una pequeña parte, sobre los propios paramentos verticales.

La Coruña, al estar situada en una latitud de 43022 ' recibe una radiación solar anual, sobre superficie vertical orientada al sur, de las más bajas de España, del orden de $60.500 \mathrm{~W} / \mathrm{m}^{2} \mathrm{y}$, 
por lo tanto, en época de invierno, cuando la temperatura exterior registra valores medios de unos $10 \circ \mathrm{C}$, se necesitan sistemas que permitan una gran captación de calor para poder elevar su temperatura interior. En época estival, como la temperatura exterior media es de unos $19 \circ \mathrm{C}$, muy próxima a la temperatura del confort, será necesaria la intervención del usuario para que, mediante la apertura de las galerias de la fachada, permita una gran circulación de aire anulándose el efecto invernadero y, por otro lado, la elevación de la temperatura interior.

A medida que nos traslademos hacia el sur, principalmente en microclimas donde el salto térmico a lo largo del día es amplio, pudiendo alcanzar en algunas zonas valores de unos $20 \circ \mathrm{C}$, las construcciones del lugar experimentan un cambio de fisonomia, pudiendo apreciarse que sus cerramientos están constituidos a base de mamposteria de piedra granitica de gran espesor, con superficies de acristalamiento de valores medios, provocando de esta forma una gran acumulación térmica en los cerramientos mientras exista radiación solar y aprovechando este calor acumulado para ir cediéndolo, tanto al ambiente exterior como al interior, con un determinado desfase cuando los niveles térmicos sean inferiores a los del cerramiento. De forma análoga podria decirse en el momento que los cerramientos, durante el periodo nocturno, prácticamente igualan su temperatura con la del ambiente externo, con lo cual durante el dia elevan su nivel térmico a costa de la captación solar, así como de la energía térmica recibida desde el ambiente interior.

Con este tipo de cerramientos, el ambiente interior a las fuertes oscilaciones de temperaturas exteriores, entre el dia y la noche, no se producirá de forma inmediata, actuando los cerramientos como masa reguladora térmica.

Si extrapolamos el sistema indicado anteriormente nos encontramos que a medida que la inercia térmica del cerramiento vaya creciendo, el intervalo de temperatura interior irá disminuyendo; por lo tanto, lo más sencillo para el mismo material es aumentar el espesor del cerramiento, con lo que se eleva simultáneamente la resistencia térmica y la capacidad térmica, llegándose a espesores enormes.

También tiene especial influencia sobre la inercia térmica, la cantidad de masa acumulada en el interior, así como su distribución. Sobre este aspecto pueden apreciarse los trabajos efectuados en el IETcc sobre unos módulos de diferente inercia térmica con la misma masa interna, pero con una distribución distinta, bien sea a lo largo de toda la periferia sobre un (c) Consejo Superior de Investigaciones Científicas Licencia Creative Commons 3.0 España (by-nc) ángulo, o concentrada en la zona central, y que están recogidas en el libro «Bases para el diseño solar pasivo» (4).

En lineas generales puede decirse que, para edificaciones de tipo medio y pesadas, la influencia del mobiliario y demás elementos existentes en el interior de la vivienda, sobre el indice térmico global, viene a ser del $5 \%$.

Sin embargo existen otros casos, de tipo industrial principalmente, opuestos al interior, donde la influencia de la masa concentrada en su interior es tan notable que prácticamente puede despreciarse el porcentaje acumulado al indice térmico global, debido al cerramiento y cubierta.

Uno de los casos fue analizado en el IETcc, realizándose un estudio sobre un proyecto de construcción de unas bodegas, con gran cantidad de vino acumulada en su interior y distribuido en la zona central de tal forma que, la variación de temperatura máxima que debia experimentar el vino, estuviese dentro de unos determinados márgenes. En estas

circunstancias, como la influencia de la masa era tan importante con respecto al cerramiento periférico y cubierta, se optó por el diseño de un cerramiento de tipo ligero que, prácticamente, sólo tenia como finalidad fundamental evitar la radiación solar directa, que podria provocar una elevación puntual de temperaturas superiores por las tolerancias indicadas para su correcta conservación.

Dentro de nuestra amplia gama de arquitectura popular existen algunos tipos de construcciones que elevan al máximo el criterio de inercia térmica, incorporados dentro de una arquitectura subterránea, donde las viviendas están integradas en el propio relieve

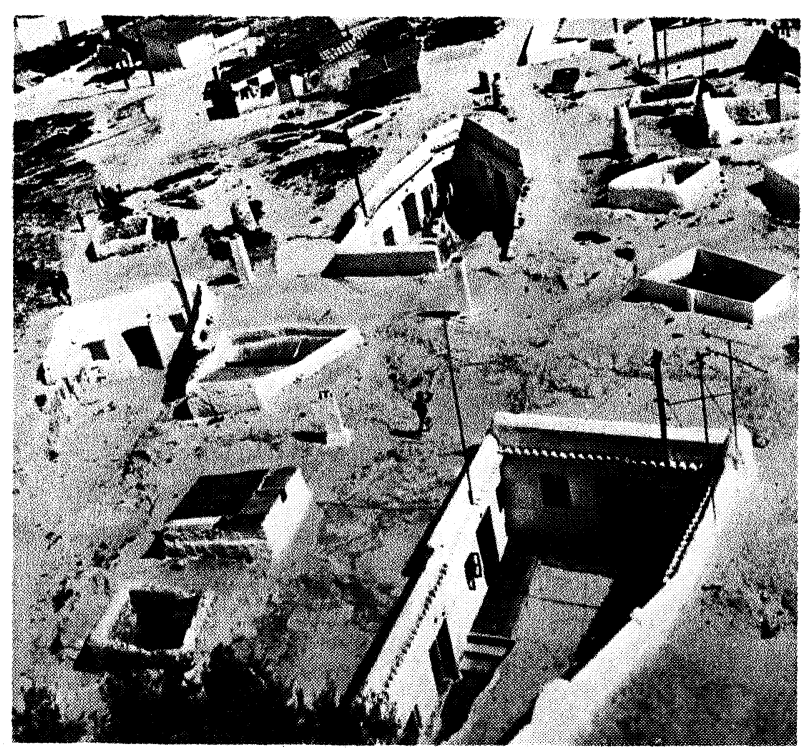

Foto 2

http://informesdelaconstruccion.revistas.csic.es 


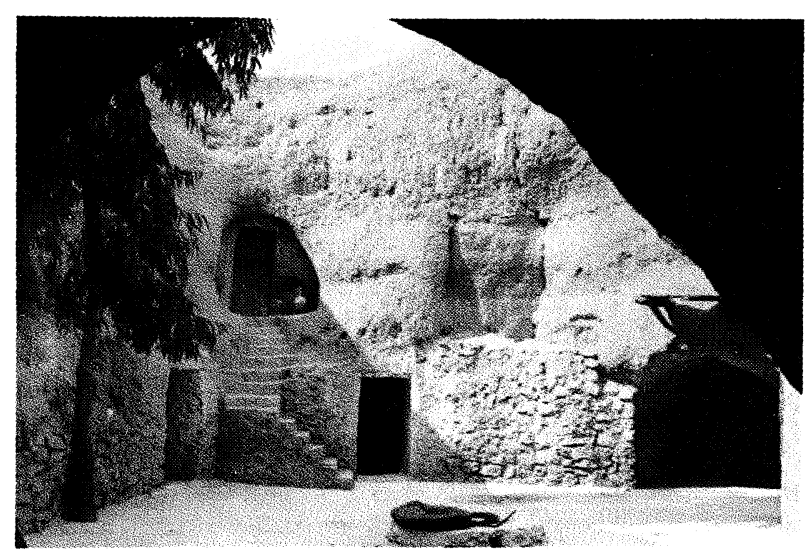

Foto 3

montañoso, o bien en el subsuelo, aunque por desgracia este tipo de edificaciones tiende a desaparecer, tal como ocurrió con las viviendas de Benimanet (Foto 2).

Recientemente, y en época de verano, he tenido la oportunidad de permanecer durante algunos dias en este tipo de edificaciones, situadas en las montañas del sur de Túnez, en los poblados de Matmata, foto 3 , donde las viviendas estaban realizadas de forma subterránea a una profundidad variable entre 3 y 8 metros. El clima de esta región es cálido y seco, llegando a alcanzar con frecuencia temperaturas de 50 a $550 \mathrm{C}$, en la época de verano, cuando en las habitaciones interiores las temperaturas alcanzan valores de $280 \mathrm{C}$, permaneciendo dentro de un intervalo de $30 \mathrm{C}$ durante varios dias, es decir, a esas profundidades del terreno puede suponerse que los cambios térmicos producidos por el ambiente exterior no influyen prácticamente en las condiciones térmicas internas.

Pudiera parecer que estas viviendas, al estar por debajo de la cota \pm 0 del terreno, carecerian de todo tipo de iluminación fundamental para poder lograr un buen ambiente fisico; sin embargo, debido a la disposición de las distintas viviendas alrededor de un patio central, de unos $50 \mathrm{~m}^{2}$, permite recibir una gran radiación solar a través de la puerta y ventana que, prácticamente, ilumina toda la vivienda.

Volviendo de nuevo a la Geografia española hacia el sur se aprecia otro paisaje completamente distinto a consecuencia de los llamados "Pueblos blancos de Andalucia" que, a la inversa de lo indicado para la zona gallega, poseen gran abundancia de radiación solar, por lo que sobre superficie vertical orientada al sur llega a ser del orden de $85.400 \mathrm{~W} / \mathrm{m}^{2}$, lo cual representa un incremento de un $30 \%$ en relación con el otro caso, donde las temperaturas ambientales de confort térmico se aproximan más a los meses de invierno, generalmente.

Como efecto de esta climatologia, la arquitectura popular (3) se ha desarrollado por otros cauces muy diferenciados, tendentes de forma principal a disminuir la radiación incidente en las viviendas.

Tratando de evitar esta radiación solar, puede observarse su bajo porcentaje de superficies acristaladas, del orden del $10 \%$, y en muchos casos, principalmente en época estival, suelen emplearse elementos protectores, como persianas o parasoles.

La incidencia directa o indirecta de la radiación solar, sobre los cerramientos, lleva aparejados una serie de procesos, tales como: elevación de la temperatura superficial exterior, calentamiento del cerramiento, cesión de calor al ambiente interior y, por último, elevación de la temperatura interior. Todos estos fenómenos están en función de las caracteristicas del cerramiento y disposición de los materiales.

Por lo tanto, la tendencia para conservar una temperatura interior, en la que tenga poca influencia la del ambiente, es conseguir que la temperatura sol-aire, o temperatura de una superficie expuesta al sol, sea lo más próxima a la del ambiente, por lo cual nos fijaremos en la fórmula siguiente:

$$
T_{s a}=T_{a}+\frac{1}{h_{e}}\left(d I_{t}-\varepsilon I_{l}\right)
$$

donde:

Tsa $=$ Temperatura sol-aire (o C)

$\mathrm{Ta}=$ Temperatura ambiente exterior (o C)

$I_{\mathrm{t}} \quad=$ Intensidad total (directa + difusa) de radiación solar incidente sobre la superficie exterior $\left(\mathrm{W} / \mathrm{m}^{2}\right)$

$I_{1}=$ Radiación de larga longitud de onda de una superficie negra a temperatura ambiente exterior (Para cubiertas horizontales alcanza un valor de 100 $\mathrm{W} / \mathrm{m}^{2}$ con cielo nublado. En el caso de superficies verticales, se considera que la larga radiación ganada por el terreno equivale a la pérdida hacia cielo, y por lo tanto $I_{1}=0$ )

$h_{e}=$ Coeficiente superficial externo de transmisión de calor $(\mathrm{W} / \mathrm{m} \circ \mathrm{C})$

$\alpha=$ Coeficiente de absorción de radiación solar de la superficie exterior (depende de las caracteristicas superficiales, tales como color, rugosidad, brillantez, etc...)

$\varepsilon \quad=$ Emisión de la superficie exterior de larga longitud de onda 
Teniendo en cuenta las consideraciones para suferficies verticales, indicadas anteriormente, habrá que actuar sobre el segundo término disminuyendo $I_{t}$ y $\alpha$, y aumento el coeficiente superficial externo de transmisión de calor $\left(\mathrm{h}_{\mathrm{e}}\right)$.

Esta disminución de $\mathrm{I}_{\mathrm{t}}$ puede observarse en gran número de pueblos en que se ha conseguido, mediante una ordenación urbanistica de los vecinos, ir edificando las viviendas con una cierta proximidad, con objeto de producir una sombra arrojada que evite la radiación sobre la superficie exterior, independientemente de otras acciones que llevan voluntariamente los usuarios con la incorporación de protecciones solares.

No obstante, como es muy dificil conseguir evitar la radiación en todo el paramento, para disminuir ésta habrá que adoptar valores del coeficiente de absorción $(\alpha)$, bajos, a base de pinturas en colores claros, fundamentalmente el blanco, y con superficies lisas $(\alpha \simeq 0,2)$, en comparación con otros materiales tradicionales, como ladrillos, piedra granitica, mortero de cemento etc..., cuyos valores oscilan alrededor del 0,8

Otro factor que influye notablemente es el coeficiente superficial externo de transmisión de calor, para el caso de convección natural en cerramientos verticales y flujo horizontal adoptándose el valor de $h_{e}=17 \mathrm{~W} / \mathrm{m}^{2} \circ \mathrm{C}$. Si se cambian las condiciones de flujo laminar a flujo turbulento mediante el establecimiento de corrientes de aire, en virtud de una adecuada orientación de las fachadas, con relación a los vientos dominantes del lugar, el factor $h_{e}$ adquirirá valores más elevados al considerar que se origina flujo turbulento $\left(\mathrm{N}_{\mathrm{Gr}} / \mathrm{N}_{\mathrm{Re}^{2}}<1\right)$, y la fórmula simplifica da seleccionada, de las indicadas por diversos autores, es:

$$
R_{e}=\frac{1}{\varepsilon h_{r}+h_{c}}
$$

donde:

$R_{e}=$ Resistencia técnica superficial exterior $\left(\mathrm{m}^{2} \circ \mathrm{C} / \mathrm{W}\right.$

$\varepsilon \quad=$ Emisión de la pared a temperatura normal de radiación. Para los cerramientos considerados puede suponerse un valor de 0,85

$h_{r} \quad=$ Coeficiente de radiación. Para la mayoria de los materiales de construcción a $200 \mathrm{C}$, tiene un valor de $5,7 \mathrm{~W} / \mathrm{m}^{2}$ o $\mathrm{C}$ )

$h_{c}=$ Coeficiente de convección. Para superficies bañadas por flujo de aire a la velocidad $v$, se puede aplicar la fórmula: $h_{c}=5,8+4,1 \vee W / m^{2} \circ C$
Si consideramos velocidades del orden de 2,5 $\mathrm{m} / \mathrm{s}$, podremos aceptar un valor $h_{e}=\frac{1}{R_{e}}=21$ $\mathrm{W} / \mathrm{m}^{2} \circ \mathrm{C}$.

Como puede apreciarse, este valor es significativamente superior al considerado con régimen laminar $\mathrm{y}$, por lo tanto, también tendrá gran influencia en la temperatura del ambiente interior.

Analizando las estructuras urbanisticas de los distintos ejemplos considerados de arquitectura popular, puede apreciarse que disponen, generalmente, de un denominador común: de expansión de las poblaciones al conseguir que las fachadas principales tengan una orientación sur y, por lo tanto, reciban la máxima radiación solar en invierno y minima en verano -según puede apreciarse en la Tabla I-, en tres latitudes significativas, durante los meses de enero y junio, según datos obtenidos de radiación solar sobre superficies inclinadas (5).

\section{TABLA I}

Radiación Global Incidente $\left(\mathrm{W} / \mathrm{m}^{2}\right)$ sobre superficie vertical, orientación Sur

\begin{tabular}{|c|c|c|}
\hline Latitud & Enero & Junio \\
\hline 430 & 5.600 & 4.530 \\
$40^{\circ}$ & 6.120 & 5.470 \\
370 & 8.260 & 5.030 \\
\hline
\end{tabular}

\section{ARQUITECTURA BIOCLIMATICA}

La entrada de tendencias innovadoras en la edificación hizo que se olvidara, casi por completo, la historia que se venia arrastrando a través de las fuentes arquitectónicas y que estaba fuertemente ligada con el propio desarrollo socio-económico-cultural de los pueblos, dándoles un carácter especial y diferenciado para ir incorporando una fisonomia de edificación totalmente impersonal que, en muchos casos, estaba en contraposición con el sentido de bienestar interior, pero que era suplido mediante el aporte de energia por otros medios técnicos para poder conseguir unas condiciones climáticas internas más próximas a las del confort.

Con la crisis energética se crea un fenómeno de inversión volviendo al estudio y significación mediante razonamientos técnicos de los condicionantes que afectaban, a los antiguos moradores, a diseños constructivos adecuados a las condiciones ambientales.

Como respuesta a esta actuación divergente motivada por un ahorro energético surge una 
arquitectura nueva, una arquitectura que pudiéramos llamar Bioclimática, estableciendo una interacción entre el hombre, clima y región tendente a obtener un hábitat con unas condiciones de confort óptimas, mediante la máxima utilización de los recursos geográficos, materiales y condiciones climáticas propias de la zona, considerando conceptos termofísicos que hasta el momento actual no se utilizaban.

Un gran campo de actuación dentro de la arquitectura bioclimática, se encuentra en el aprovechamiento mediante sistemas pasivos de energia solar.

Toda esta arquitectura bioclimática tiene como fuente de conocimientos los distintos ejemplos prácticos que se pueden apreciar por toda la geografia española algunos de los cuales ya se han indicado anteriormente.

\subsection{Enfriamiento pasivo}

Aunque en España la diversidad de climas es abundante puede decirse que en un pais "soleado", ya que, según datos estadisticos, las horas de sol anual en las distintas estaciones metereológicas oscilan desde un valor minimo, en Oviedo, con 1.637 a un valor máximo, en Almeria, con 3.053 horas, por lo tanto, en la utilización de sistemas de captación pasiva de energia solar deberá tenerse especial cuidado, ya que aunque en invierno se puedan obtener unas condiciones internas adecuadas, si no están consideradas en el diseño, o bien no se utilizan medios pasivos de enfriamiento, con la actuación directamente del propio usuario, se pueden obtener unos sobrecalentamientos en el interior importantes creando, por lo tanto, unas condiciones internas más desfavorables que en el exterior y, como consecuencia, no se habria conseguido ningún avance ni tendria ningún interés la aplicación de la captación pasiva.

En el IETcc se ha podido comprobar, sobre cuatro módulos experimentales de $27 \mathrm{~m}^{3}$ de volumen, con sistemas de captación solar pasiva (Foto 4) y constituidos por muro Trombe efecto invernadero, captación directa y efecto invernadero con sistema de acumulación mediante lecho de piedras; la elevación de la temperatura interior en época de invierno se encuentra por encima de los limites de confort establecidos llegando, incluso, a alcanzar valores de $380 \mathrm{C}$ para el caso de radiación directa.

Por lo tanto, se hace necesaria una actuación dentro de este campo bioclimático del enfriamiento pasivo, que en el momento actual tiene una baja consideración dentro del diseño arquitectónico.

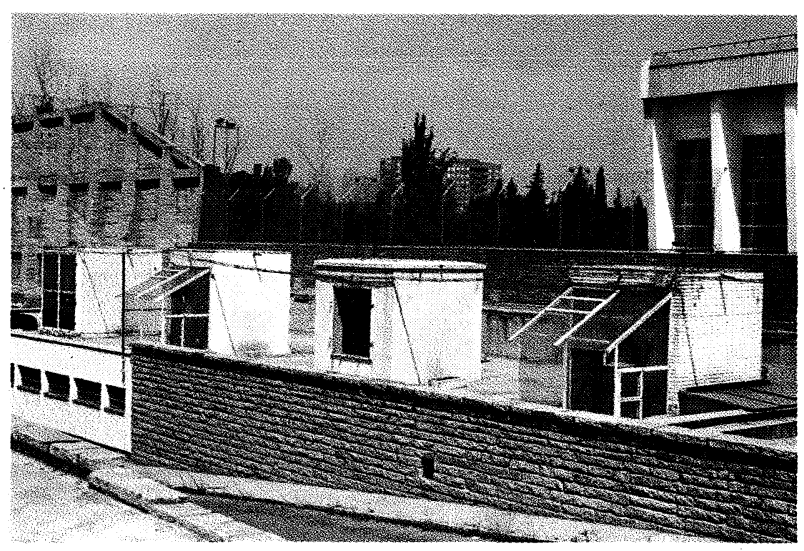

Foto 4

Foto: J. J. López del Amor. IETcc/CSIC.

\subsubsection{Sistemas de enfriamiento pasivo}

El enfriamiento pasivo lo podemos entender bajo dos aspectos diferenciados tendentes ambos a acercar al usuario a unas condiciones ambientales de bienestar. El primero por medio de evitar o disminuir el aporte térmico producido por la implantación de los distintos sistemas de captación solar pasiva; tal es el caso, por ejemplo, de la supresión de los efectos invernadero en las galerias acristaladas, o bien la producción de sombras que eviten la radiación directa. El segundo aspecto a contemplar son los posibles métodos utilizados para el enfriamiento interior de las viviendas, provocados por el propio diseño arquitectónico $\mathrm{u}$ otros mediante la acción del propio utilizador.

Algunos de estos procedimientos son de gran utilización dentro de nuestra geografia, los cuales han sido considerados en algunos de los ejemplos enumerados anteriormente.

Los tres grandes bloques en que podemos agrupar a los diferentes sistemas son los siguientes:

1. Fenómenos convectivos ambientales.

2. Emisión de radiación.

3. Aprovechamiento del nivel térmico del subsuelo.

A continuación analizaremos, de forma más amplia, cada uno de estos tres apartados:

\subsubsection{Fenómenos convectivos ambientales}

Están basados fundamentalmente en el aprovechamiento de los vientos dominantes del lugar o la creación de fenómenos convectivos que provoquen una abundante pérdida de calor, tanto en la estructura como en el interior de la vivienda. 
El calor cedido estará regido por la ley de Newton, de acuerdo con la fórmula siguiente:

$$
\mathrm{q}=\mathrm{h}_{\mathrm{c}} \mathrm{A}(\Delta \mathrm{T})
$$

donde:

$\mathrm{h}_{\mathrm{c}}=$ coeficiente de convección $\left(\mathrm{W} / \mathrm{m}^{2} \circ \mathrm{C}\right)$

$A=$ superficie de pérdida $\left(\mathrm{m}^{2}\right)$

$\Delta \mathrm{T}=$ salto térmico $(\mathrm{O} \mathrm{C})$

A medida que se aumenta el valor de $h_{c}$, se origina una mayor cesión de calor, aspecto éste que ya fue recogido en el apartado 2.1.

La localización y disposición de los edificios, es decir, el planteamiento urbanistico del entorno, es fundamental para una modificación de los fenómenos convectivos que inciden sobre los edificios, pudiéndose provocar anomalias aerodinámicas con la creación de corrientes turbulentas. Estas perturbaciones dan lugar a determinados efectos, entre los que podriamos citar como más significativos los siguientes: efecto esquina, efecto rodillo; etc... (6).

Con el fin de incrementar la acción del viento y favorecer los diversos efectos perturbadores, en muchos pueblos de Andalucia, tales como Casares, Mijas, Mojácar, Salobreña, etc..., puede apreciarse su disposición agrupados en colinas, donde se provocan áreas de gran velocidad, sobre todo en las costas.

Indudablemente el efecto de refrigeración será más acusado en el periodo nocturno y solamente tendrá efectividad cuando las superficies externas de los cerramientos y cubiertas sean de colores claros. En caso contrario, cuando sean de colores oscuros, la temperatura interior durante el día alcanzará valores superiores a los del ambiente exterior y por lo tanto resultará imprescindible durante el dia ventilar para que se produzca una renovación del aire interior más caliente que el exterior.

Los mecanismos por los que se rige esta sustitución están basados en la combinación de los siguientes factores: a) condiciones del

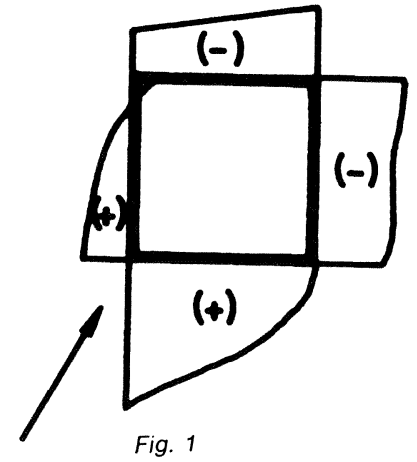

viento alrededor del edificio; b) el efecto del sistema de ventilación interior y c) la diferencia de temperaturas entre el ambiente interior y exterior, lo que crea una presión diferencial entre las distintas superficies del edificio (figura n.o 1), dando lugar a una ventilación cruzada.

Los criterios de funcionamiento de esta ventilación se rigen por lo establecido en el teorema de Bernoulli, de acuerdo con la siguiente expresión:

$$
\frac{\mathrm{P}}{\omega}+\frac{\mathrm{V}^{2}}{2 \mathrm{~g}}+\mathrm{z}=(\text { diferencial } e)
$$

donde:

$$
\begin{aligned}
& \mathrm{P}=\text { presión absoluta }\left(\mathrm{kp} / \mathrm{m}^{2}\right) \\
& \mathrm{v}=\text { velocidad del aire }(\mathrm{m} / \mathrm{s}) \\
& \mathrm{z}=\text { altura geométrica }(\mathrm{m}) \\
& \omega=\text { peso especifico }\left(\mathrm{kg} / \mathrm{m}^{3}\right)
\end{aligned}
$$

En el aprovechamiento de la ventilación cruzada tiene un papel importante la distribución de la tabiqueria interior, pudiendo obtener un recorrido del aire adecuado por el interior de la vivienda.

Cuando en la vivienda existe una comunicación con el exterior en las zonas elevadas, la diferencia de temperaturas entre el interior y el exterior ocasiona una presión diferencial que da lugar al denominado "efecto chimenea", debido a la diferencia de densidades del aire a distintas temperaturas. Este efecto causa un vacio en las partes inferiores del edificio y un exceso de presión en las zonas altas, por lo tanto a una cierta altura existe una zona neutra, donde las presiones internas y exteriores son las mismas. Esta presión diferencial está dada por la expresión siguiente:

$$
P=9,8 \cdot \rho \cdot h\left(1-\frac{T_{e}}{T_{i}}\right)
$$

donde:

$\mathrm{P}=$ presión diferencial $\left(\mathrm{P}_{\mathrm{a}}\right)$

$\rho=$ densidad del aire $\left(\mathrm{kg} / \mathrm{m}^{3}\right)$

$T_{i}=$ Temperatura ambiente interior $(\mathrm{O} C)$

$\mathrm{T}_{\mathrm{e}}=$ Temperatura ambiente exterior $(\mathrm{O} \mathrm{C})$

$\mathrm{h}=$ distancia a la zona neutra $(\mathrm{m})$

La ventilación mediante fenómenos convectivos parece idónea en aquellas regiones donde exista un gran salto térmico entre el dia y la noche, ya que durante el periodo nocturno toda la estructura está cediendo gran cantidad de calor a la atmósfera y permite una mejor 
estancia de los moradores durante el dia. Este efecto es más acusado cuando se habla de cerramientos de gran inercia térmica.

Sin embargo, cuando se trata de regiones cálidas, donde el salto térmico no es importante y la temperatura del ambiente exterior es algo inferior a la del confort, la pérdida térmica por la estructura de la vivienda es inferior, no llegándose, en muchos casos, a poder emplearse como una refrigeración efectiva; por lo tanto, se habrán de incorporar otros factores tendentes a incrementar las pérdidas térmicas.

Para aumentar las pérdidas térmicas, de acuerdo con la ecuación (3), habrá que actuar fundamentalmente sobre los factores siguientes:

a) Incremento de la superficie de pérdida. Este incremento de superficie deberá estar situado en la zona interna de la vivienda, para lo cual se podrán efectuar ondulaciones, ranuras, etc., consiguiéndose a la vez una superficie más homogénea térmicamente. La mayor cesión del calor al interior deberá estar intimamente ligada con una eficaz ventilación interior. También puede acelerarse el desprendimiento de calor por la superficie estructural mediante la circulación del aire por conductos verticales efectuados en la propia masa estructural.

b) Elevación de las corrientes de convección por las superficies. Es necesario que las entradas de aire se orienten principalmente al producir un baño de las superficies que acumulan calor durante el periodo diurno, para aumentar el coeficiente superficial de convección interna y, en especial, hacia el techo, donde se acumulan las capas más cálidas de aire, dando lugar a un efecto termofísico en todo el volumen de la habitación, provocándose la renovación de aire con el exterior.

Otro sistema para activar la circulación convectiva, en el interior de la vivienda, se puede conseguir a través de un lecho de piedras situadas al abrigo de la radiación solar y bien aisladas térmicamente que, durante la noche, una vez retirado el aislamiento, se somete el lecho de piedras a un rociamiento con agua, con lo cual se origina una evaporación generalizada disminuyendo su temperatura, para que en el periodo diurno, mediante convección natural o forzada, sea introducido aire al ambiente interior, o bien mediante sistemas de conductos por interior de la propia estructura.

\subsubsection{Emisión de radiación}

Está basada en la energia, en forma de radiación de onda larga, que emiten todos los cuerpos, aunque al mismo tiempo reciba parte de radiación de la atmósfera, siendo la diferencia entre ambas la que se aprovecha con fines de refrigeración.

En un edificio este intercambio radiante es más acusado en la cubierta, donde se puede considerar al cielo como una superficie envolvente, teniendo un factor en forma de 1 , en comparación con las superficies verticales, donde el factor de forma puede considerarse 0,5 .

La ley general sobre la que se fundamentan estos fenómenos de intercambio térmico entre dos superficies a temperaturas $T_{1}, T_{2}$-una de las cuales envuelva completamente a otraviene dada por la ley de Stefan-Boltzmann, que tiene por expresión la siguiente:

$$
q=\varepsilon \cdot \sigma \cdot\left(T_{1}^{4}-T_{2}^{4}\right)
$$

donde:

$\varepsilon=$ Emisividad de la superficie 1

$r$ = Constante de Stefan-Boltzmann $\left(5,67 \cdot 10^{-8} \mathrm{~W} / \mathrm{m}^{2} \cdot \mathrm{K}^{4}\right)$

$\mathrm{T}_{1}$ y $\mathrm{T}_{2}=$ Temperaturas de las superficies $1 \mathrm{y}$ 2 respectivamente $(\circ \mathrm{K})$

El espectro de radiación electromagnética emitida por el sol, abarca un intervalo de longitudes de onda de 0,3 a $50 \eta \mathrm{m}$ (figura $\mathrm{n} .{ }^{\circ}$ 2), no obstante, la principal energia térmica queda dentro de la gama de corta longitud de onda, según los porcentajes de la tabla siguiente; considerada para un dia de claro:

\begin{tabular}{|c|r|}
\hline $\begin{array}{c}\text { ENERGIA SOLAR } \\
(\%)\end{array}$ & $\begin{array}{c}\lambda \\
(\mu \mathrm{m})\end{array}$ \\
\hline 95 & $0,3-2,4$ \\
1,2 & 0,3 \\
3,6 & 2,4 \\
0,05 & $4-50$ \\
\hline
\end{tabular}

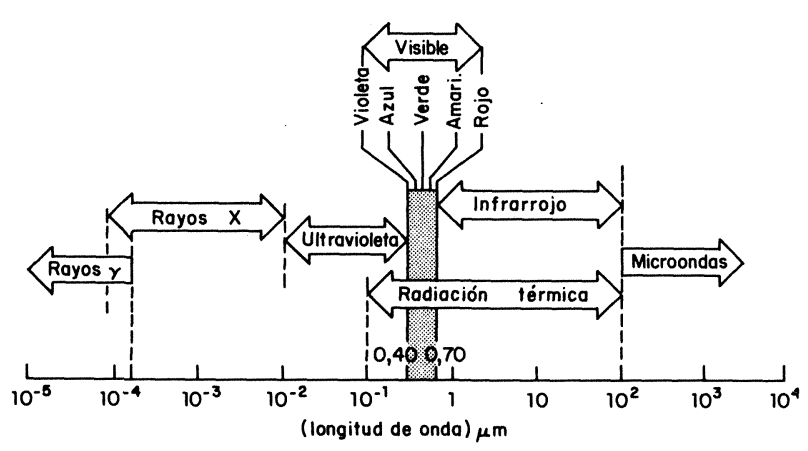

Fig. 2 
La irradiación que llega a una superficie queda disminuida por unos coeficientes de atenuación y turbiedad de la atmósfera, siendo los más significativos los originados por el efecto de las nubes y la humedad del ambiente.

En el periodo nocturno la emisión de radiación de onda larga se hace más significativa y estará en función de las temperaturas del cielo y del emisor.

La temperatura del cielo claro está dada por la expresión:

$$
T_{c}=\left(\varepsilon_{c}^{0,25}\right) T_{a}
$$

donde:

$$
\begin{aligned}
& T_{c}=\text { temperatura del cielo }(\circ \mathrm{K}) \\
& T_{a}=\text { temperatura del ambiente }(0 \mathrm{~K}) \\
& \varepsilon_{c}=\text { emisividad del cielo }
\end{aligned}
$$

Para el cálculo de la emisividad del cielo, se han establecido diversas fórmulas empiricas tales como:

$$
\begin{aligned}
& \varepsilon \mathrm{C}=0,66+0,40 \mathrm{P}_{\mathrm{v}}^{1 / 2} \text { (Kondratyev) } \\
& \varepsilon \mathrm{C}=0,787+0,0028 \mathrm{t}_{\mathrm{r}} \text { (Clark y Allen) } \\
& \varepsilon \mathrm{C}=0,741+0,0062 \mathrm{t}_{\mathrm{r}} \text { (Berdahl y Fromberg) }
\end{aligned}
$$

donde:

$$
\begin{aligned}
t_{r} & =\text { temperatura de rocio }(\circ \mathrm{C}) \\
\mathrm{P}_{\mathrm{v}} & =\text { presión parcial de vapor (milibares) }
\end{aligned}
$$

cuya representación puede observarse en la figura n.o 3.

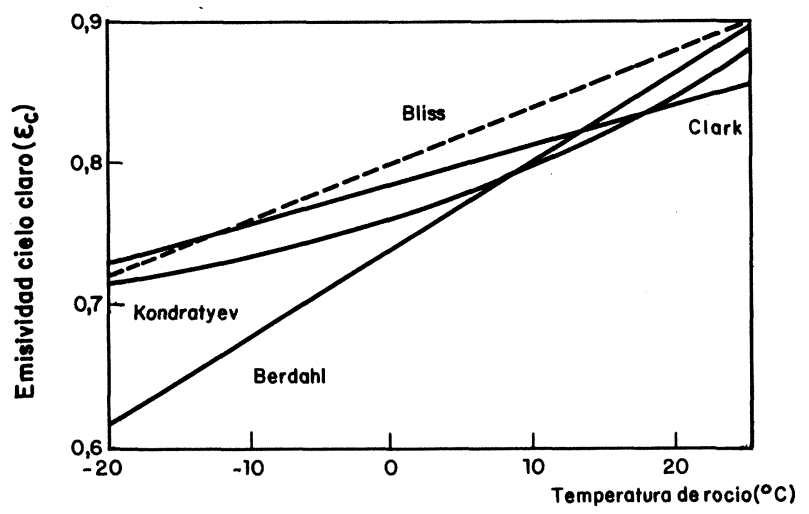

Fig. 3

Indudablemente, como en un edificio el emisor más importante es la cubierta, su intercambio de radiación tendrá por valor:

$$
\begin{gathered}
\mathrm{R}_{\text {neta }}=\mathrm{R}_{\text {emitida }}-\mathrm{R}_{\text {absorbida }} \\
\mathrm{R}_{\text {neta }}=\sigma \cdot \varepsilon_{\mathrm{r}} \cdot \mathrm{T}_{\mathrm{c}}^{4}-\sigma \cdot \alpha_{\mathrm{r}} \cdot \mathrm{T}_{\mathrm{c}}^{4}
\end{gathered}
$$

Sustituyendo los valores obtenidos antes y teniendo en cuenta que para esa gama de espectro de radiación de onda larga $\alpha_{\mathrm{r}}=\varepsilon_{\mathrm{r}}$ tendremos:

$$
\begin{aligned}
\mathrm{R}_{\text {neta }}= & \sigma \cdot \varepsilon_{\mathrm{r}} \cdot \mathrm{T}_{\mathrm{a}}^{4}-\sigma \cdot \varepsilon_{\mathrm{r}} \cdot \varepsilon_{\mathrm{c}} \cdot \mathrm{T}_{\mathrm{a}}^{4}=\sigma \varepsilon_{\mathrm{r}} \\
& \left(\mathrm{T}_{\mathrm{r}}^{4}-\left[0,741+0,0062 \mathrm{t}_{\mathrm{r}}\right] \mathrm{T}_{\mathrm{a}}^{4}\right)
\end{aligned}
$$

Además de los fenómenos radiantes que se producen también llevan incorporados un intercambio convectivo entre la superficie radiante y el ambiente, que irán disminuyendo a medida que se vayan equilibrando ambas temperaturas, llegando incluso a ser desfavorables y produciendo ganancia de calor del ambiente cuando la temperatura del radiador haya descendido por debajo de la del ambiente. Por lo tanto, es necesario que las cubiertas posean una gran capacidad térmica y sirvan continuamente de radiador de onda larga.

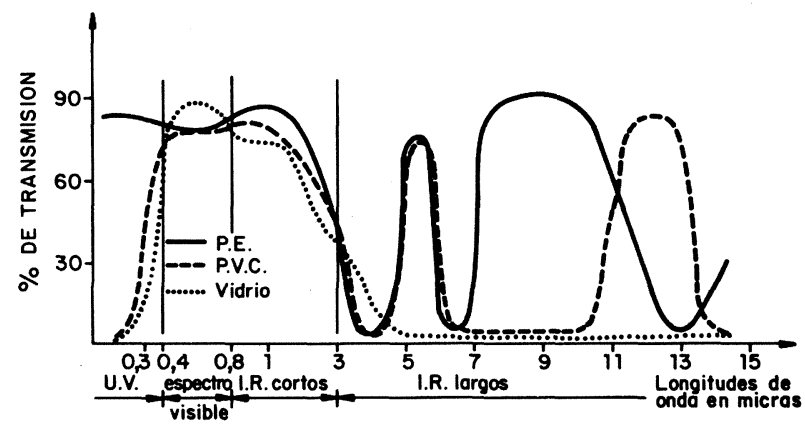

Fig. 4

Cuando los radiadores son de peso ligero -se han realizado estudios experimentales en Israel (7)- mediante la utilización de láminas de polietileno transparente, en un 60 u $80 \%$ a la radiación de onda larga (figura n.o 4), e incorporándola firmemente al radiador, da lugar a la creación de una capa de aire frio inmóvil por encima del radiador, alcanzando de este modo temperaturas más bajas de la superficie radiante y evitando el vapor térmico por fenómenos convectivos.

Recientes estudios están tratando de la incorporación de superficies selectivas, de forma que tengan una gran emisividad y poder de absorción, dentro de la gama de 8 a 13 micras (región de transmisiones atmosféricas) y una gran reflectividad en el resto del espectro.

\subsubsection{Aprovechamiento del nivel térmico del subsuelo}

Como ya se indicó anteriormente al hablar de la arquitectura subterránea, el exponente más claro puede encontrarse en los pablados de 
Matmata, donde se produce un aprovechamiento eficiente de las caracteristicas tèrmicas del subsuelo.

El proceso de transmisión térmica que se produce es el de conducción sobre un sólido semi-infinito de la onda térmica ambiental (8), a través del suelo, dando como resultado las distintas temperaturas en función de la profundidad, de acuerdo con la expresión siguiente:

$$
T_{(z, t)}=T_{m}-A_{s} \cdot \varepsilon^{-z \eta} \cos \left[\frac{2 \pi}{365}\left(t-t_{o}\right)-z \eta\right]
$$

donde:

$T_{(z, t)}=$ Temperatura del suelo a la profundidad $x$ y tiempo t (o C)

$T_{m}=$ Temperatura media anual del suelo (O C)

$A_{s}=$ Amplitud de la onda de temperatura en la superficie del suelo (o $\mathrm{C}$ )

$\mathbf{z}=$ Profundidad $(\mathrm{m})$

$\mathrm{t}=$ Tiempo desde el comienzo (dias)

$t_{0}=$ Constante de fase (dias)

$$
\begin{aligned}
& \eta=\sqrt{\frac{\pi}{365 \cdot \alpha}} \\
& \alpha=\text { Difusividad del suelo }\left(\mathrm{m}^{2} / \mathrm{dia}\right)
\end{aligned}
$$

De forma análoga a lo acaecido en un cerramiento, al incidir la onda térmica ambiental en los distintos niveles del subsuelo, se aprecian los fenómenos de amortiguamiento y desfase de la onda térmica incidente.

La temperatura en la superficie del suelo, que es fundamental en el transporte térmico a las capas más inferiores, puede modificarse y disminuirse con relación a las zonas próximas por diversos métodos, tales como riego controlado -produciendo evaporación-, creación de sombras, etc...; de esta forma el intercambio térmico con el exterior, entre la ganancia de calor y la pérdida a través de la superficie, serä más reducida.

Como es previsible suponer las caracteristicas propias del subsuelo influyen notablemente en la temperatura y sobre todo la humedad, ya que los valores de difusividad térmica pueden elevarse dos y tres veces al valor en estado seco, debido fundamentalmente al incremento que experimenta el valor de conductividad térmica.

Considerando un supuesto de temperaturas ambientales enmarcado dentro del entorno de las temperaturas máxima y mínima diarias, a lo largo de un periodo de un año podemos ver en la figura n.o 5 las distintas evoluciones de temperaturas, tanto diarias como estacionales, que se producen en función de la profundidad que se considere.

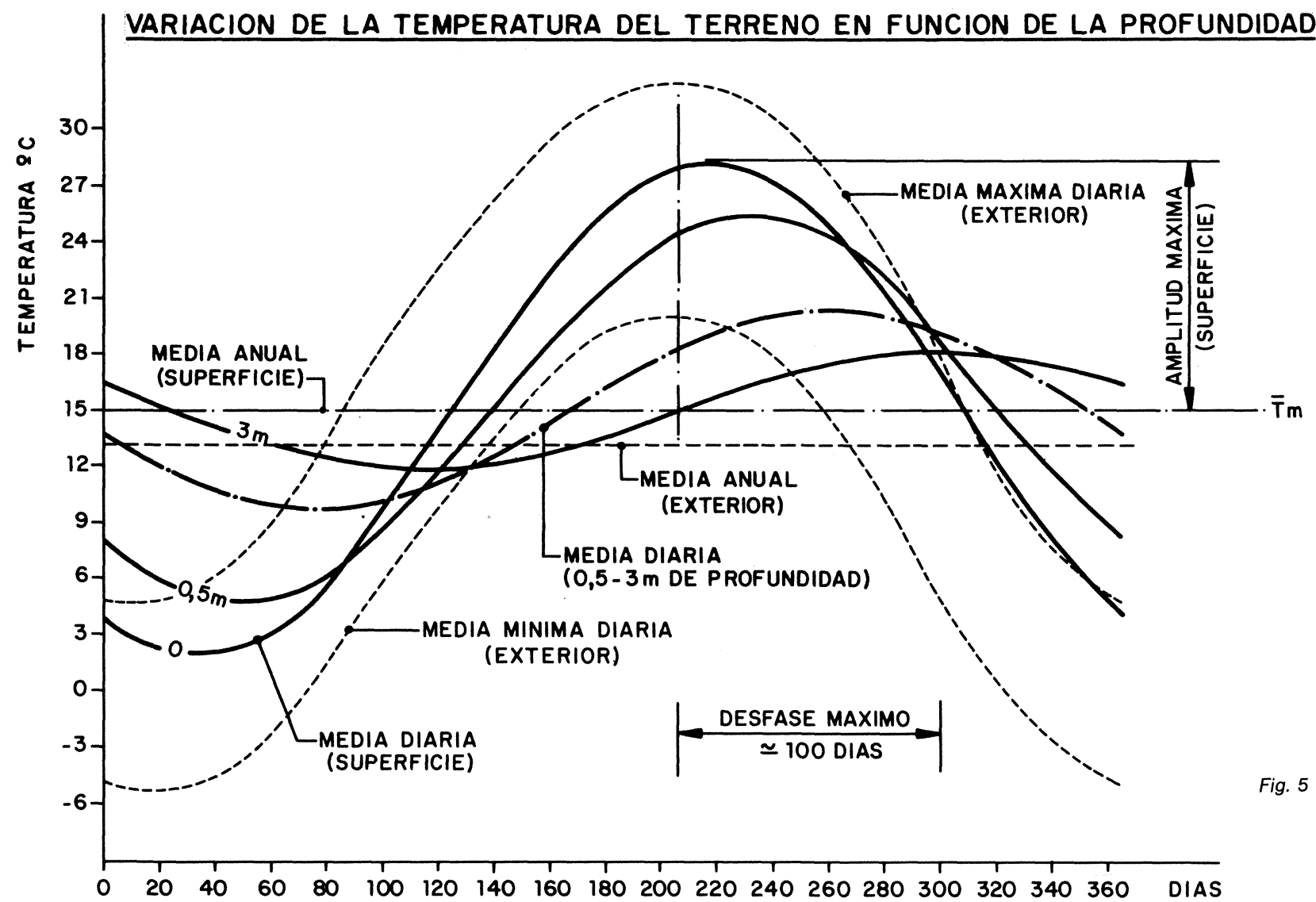


Del análisis de dicha figura puede apreciarse lo siguiente:

- A medida que aumenta la profundidad, la onda térmica es más plana y tiene un mayor desfase con relación a la temperatura exterior.

- La temperatura media anual, en la superficie, es superior a la media anual exterior en $1,5 \%$ como consecuencia de los distintos procesos de intercambio térmico que se producen, tales como absorción solar durante el dia, radiación durante la noche, fenómenos convectivos y pérdida térmica por evaporación.

- El desfase máximo estacional a una profundidad de $3 \mathrm{~m}$ es de 100 dias aproximadamente.

- Cuando en el periodo estival la temperatura ambiente oscila entre 20 y $32^{\circ} \mathrm{C}$ a una profundidad de $3 \mathrm{~m}$, la temperatura media es de $15 \circ \mathrm{C}$, mientras que en invierno la temperatura ambiente varía de -5 a $5 \circ \mathrm{C}$, y la del subsuelo a la misma profundidad toma por valor medio $160 \mathrm{C}$. Por lo tanto, el subsuelo aparece como un estabilizador térmico de las temperaturas ambientales, pudiendo emplearse tanto para los procesos de calentamiento, como de enfriamiento pasivo.

Sobre este aspecto y dentro del campo de enfriamiento pasivo, se han efectuado diversos trabajos (10) incorporando conductos a profundidad de unos 2 metros y hacer circular el aire caliente del edificio para, una vez pasado por el intercambiador del subsuelo, sea recibido de nuevo en la vivienda con un nivel térmico más reducido.

\section{BIBLIOGRAFIA}

(1) FANGER, P. O.: “Thermal Confort». Robert E. Krieger Publishing Company Malabar, Florida, 1982.

(2) (ASHRAE Standard 55-81: "Thermal Enviromenttal Conditions for Human Occupancy". Atlanta, 1981.

(3) GARCIA ARROYO, A: "Arquitectura radical, arquitectura de pueblo». Monografia n.o 318. I.E.T.C.C., 1974.

(4) GARCIA, A.; ESTEBAN, J. L.; ESCORIHUELA, M.a J.; FRUTOS, J. M.; TORROJA, B. y OLAYA, M.: "Bases para el diseño solar pasivo", C.S.I.C., 1983.

(5) RAMOS, F. y TINAUT, D.: "Radiación solar sobre superficies inclinadas». C.S.I.C., 1980.

(6) GANDENER, J. y GUYART, A.: “Integration du phénomène vent dans la conception du milien bati». Ministere de l'equipement, 1976.

(7) GIBONI, B.: «Man, climate and Architecture». Elsevier Architectural Science Series, 1969.

(8) CORSLAW, H. S. y JEAGUR, J. C.: «Conduction of Heat in solids". Osford University Press, 1978.

(9) «Passive solar: Subdivisions, Windows, Underground». American Solar Energy Society, 1983.

(10) LABS, K.: «Underground Building Climate». Solar Age, 1979.

\title{
publicación del i.e.t.c.c.
}

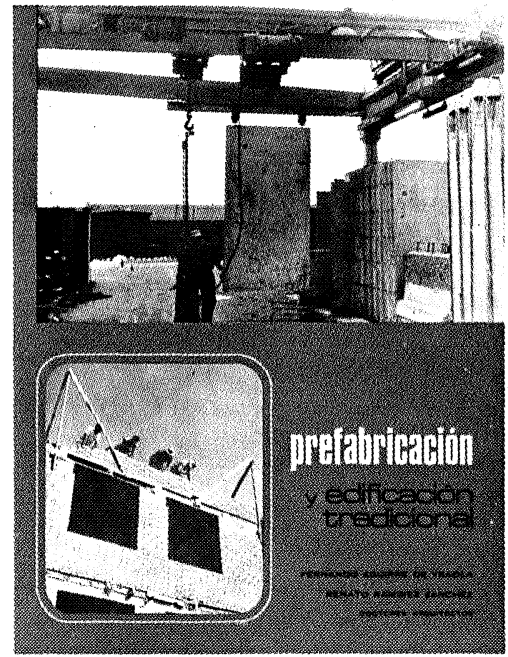

\author{
Fernando Aguirre de Yraola y Renato Ramírez Sánchez \\ Drs. Arquitectos
}

Industrialización de la construcción, en sentido general dentro de ésta, es el empleo, en forma racional y mecanizada, de materiales, medios de transporte y técnicas constructivas para conseguir una mayor productividad.

El proceso del desarrollo de la industrialización, que nos transformará el sector tradicional, o convencional, en otros más de acuerdo con nuestras exigencias actuales, deberá aplicarse no sólo al producto de este desarrollo, es decir, la edificación, sino también al conjunto de la producción.

El carácter acusadamente gráfico y esquemático de esta publicación pretende constituir una sintesis de parámetros básicos para la Racionalización, Normalización y Tipificación de elementos prefabricados, organización en obra y factoria, para el estudio económico entre construcciones convencionales e industrializadas.

Un volumen encuadernado en cartoné plastificado, de $21 \times 17 \mathrm{~cm}$, compuesto de 125 páginas, 25 cuadros y 56 figuras de linea.

Precios: España 1.500 ptas. Extranjero \$21.00. 